\title{
IDENTIFICAÇÃO DE ERROS NO PREPARO E ADMINISTRAÇÃO DE MEDICAMENTOS PELA EQUIPE DE ENFERMAGEM E DAS AÇÕES PRATICADAS PELAINSTITUIÇÃO HOSPITALAR
}

Marcus Fernando da Silva Praxedes ${ }^{1}$, Paulo Celso Prado Telles Filho

\begin{abstract}
RESUMO: Objetivou-se identificar os erros cometidos pela equipe de enfermagem, relacionados ao preparo e administração de medicamentos, bem como as ações praticadas pela instituição hospitalar onde os mesmos ocorreram. Trata-se de um estudo quantitativo descritivo, desenvolvido em uma instituição hospitalar de Minas Gerais, de que fizeram parte 35 profissionais. Constatou-se a ocorrência de 91 erros, sendo a não monitorização do paciente, após a medicação, o principal tipo, registrando $22 \%$, seguido da via de administração errada com $19 \%$. As ações mais praticadas pela instituição perante o erro foram a advertência, 35\%, seguida da não tomada de atitude com $26 \%$. Concluiu-se que os erros são quantitativamente elevados e graves e que a instituição hospitalar utiliza a advertência, que é vista como uma forma de punição, para os que cometem esses erros.
\end{abstract}

PALAVRAS-CHAVE: Enfermagem; Educação; Erros de medicação; Sistemas de medicação.

\section{IDENTIFICATION OF DRUG PREPARATION AND ADMINISTRATION ERRORS BY THE NURSING TEAM AND THE ACTIONS TAKEN BY THE HOSPITAL INSTITUTION}

\begin{abstract}
This study aimed to identify the errors committed by the nursing team, regarding drug preparation and administration, and the actions taken by the hospital where the errors occurred. This quantitative and descriptive study was developed at a hospital institution in the State of Minas Gerais. Participants were 35 professionals. Ninety-one errors were observed. The main type was not monitoring the patient after medication, with $22 \%$ occurrences, followed by inadequate administration route with $19 \%$ cases. The most common actions taken by the institution in response to the error was giving a warning, with $35 \%$ cases, followed by not adopting an attitude with $26 \%$ cases. In conclusion, errors are numerous and serious, and the hospital responds with warnings, seen as a form of punishment for those who have made such errors.

KEYWORDS: Nursing; Education; Medication errors; Medication systems.

\section{IDENTIFICACIÓN DE ERRORES EN LA PREPARACIÓN Y ADMINISTRACIÓN DE MEDICAMENTOS POR EL EQUIPO DE ENFERMERÍA Y DE LAS ACCIONES PRACTICADAS POR LA INSTITUCIÓN HOSPITALARIA}

RESUMEN: La finalidad del estudio fue identificar los errores cometidos por el equipo de enfermería, relacionados a la preparación y administración de medicamentos y también las acciones practicadas por la institución hospitalaria donde éstos ocurrieron. Se trata de un estudio cuantitativo y descriptivo, realizado en un hospital de Minas Gerais. Participaron 35 profesionales. Fueron observados 91 errores, siendo el principal tipo la falta de monitoreo del paciente posterior a la medicación, con 22\% de los casos, seguido de la vía de administración equivocada con 19\%. Las acciones más practicadas por la institución frente a los errores fueron la advertencia, con 35\%, seguida de la ausencia de actitud con 26\%. Se concluye que los errores son cuantitativamente elevados y graves y que la institución hospitalaria utiliza la advertencia, considerada como una forma de punición, para aquellos que cometen esos errores.

PALABRAS CLAVE: Enfermería; Educación; Errores de medicación; Sistemas de medicación.

${ }^{1}$ Acadêmico do Curso de Graduação em Enfermagem da Universidade Federal dos Vales do Jequitinhonha e Mucuri. Bolsista da FAPEMIG.

${ }^{2}$ Professor. Doutor. Departamento de Enfermagem. Universidade Federal dos Vales do Jequitinhonha e Mucuri.

Autor correspondente:

Marcus Fernando da Silva Praxedes

Rua Augusto de Souza Neves, 71 - 39100-000 - Diamantina-MG

Recebido: 30/05/08

E-mail:marcusfera@yahoo.com.br

Aprovado: 31/10/08

Cogitare Enferm 2008 Out/Dez; 13(4):514-9 


\section{INTRODUÇÃO}

A equipe de enfermagem faz parte do complexo sistema de medicação, que para a Joint Commission on Accreditation of Healthcare Organizations - JCAHO se divide em cinco processos: seleção e obtenção do medicamento, prescrição, preparo e dispensação, administração e monitoramento do paciente em relação aos efeitos do medicamento ${ }^{(1)}$. À equipe de enfermagem cabe a realização dos últimos processos, sendo esses atividades cotidianas e de sua responsabilidade legal ${ }^{(2)}$. Como ela atua na parte final do sistema é de fundamental importância que tenha sólidos conhecimentos para que possíveis falhas que possam ocorrer durante o processo sejam interceptadas, evitando assim que erros sejam cometidos $^{(3)}$.

Apesar do preparo e administração de medicamentos ser uma atividade cotidiana da equipe de enfermagem, percebe-se a existência freqüente de dúvidas sobre a realização correta dessas atividades ${ }^{(4)}$. Tais dúvidas podem levar o profissional a cometer erros que geram riscos graves à integridade do paciente ${ }^{(5)}$.

Assim, evidencia-se a ocorrência real de erros no preparo e administração de medicamentos, e a necessidade da orientação profissional para a realização desta prática. Nesse sentido, a orientação da equipe de enfermagem se torna uma importante medida de prevenção de erros, por ser essa a equipe que recebe a medicação, realiza o seu preparo e a administra ao paciente. Os procedimentos referentes à medicação são de responsabilidade do enfermeiro, uma vez que gerencia e orienta a equipe de enfermagem à realização desta prática, entretanto não participa ativamente dela na maioria das vezes.

A partir do exposto, cabe ao enfermeiro a orientação de sua equipe quanto ao preparo e administração de medicamentos, de modo a favorecer e proporcionar o aprendizado contínuo e/ou capacitação constante da equipe de enfermagem, no sentido de realizar esta prática com qualidade, evitando-se erros e resultados inesperados. Os momentos de capacitação, em forma de orientações individuais, oficinas, palestras, também podem ser uma oportunidade para o profissional de enfermagem compartilhar dúvidas, além da aquisição de conhecimentos que promovam qualidade às suas ações quanto ao preparo e administração de medicamentos.

Vale destacar que vários estudos discutem os tipos de erros na medicação relacionados ao preparo e administração de medicamentos, no que diz respeito a diluição, dosagem, troca de vias, de medicamentos entre pacientes e outros ${ }^{(6-11)}$. É oportuno mencionar que quando o erro grave chega a ocorrer, o profissional que o cometeu passa por uma experiência traumática ${ }^{(12)}$.

Este estudo justifica-se, portanto, pelo fato de que, a partir do momento que sejam identificados os erros relacionados ao preparo e administração de medicamentos cometidos por profissionais da equipe de enfermagem, bem como as ações praticadas pela instituição hospitalar, sejam possíveis encontrar estratégias que minimizem os riscos para os pacientes e como conseqüência, seguirá o aumento da credibilidade à Enfermagem, criando um processo mais atualizado e seguro.

Os objetivos para este trabalho foram: identificar os erros cometidos por profissionais da equipe de enfermagem relacionados ao preparo e administração de medicamentos e as ações praticadas pela instituição hospitalar em que os mesmos ocorreram.

\section{METODOLOGIA}

Trata-se de um estudo quantitativo descritivo. O método quantitativo é caracterizado tanto pelo emprego da quantificação nas modalidades referentes à coleta de dados como no seu tratamento. Nos estudos descritivos são considerados como objeto de estudo uma situação específica, um grupo ou um indivíduo ${ }^{(13)}$, buscando demostrar com exatidão a freqüência com que determinados eventos acontecem ${ }^{(14)}$.

A pesquisa foi realizada em uma instituição hospitalar beneficente de um município do interior do estado de Minas Gerais. A instituição possui 83 leitos e dentre as especialidades de atendimento de saúde estão clínica médica, ginecologia, ortopedia e cirurgia geral.

A amostra da pesquisa foi escolhida por conveniência e teve como critérios de inclusão a disponibilidade dos profissionais para responderem os questionários e a participação ativa desses no preparo e administração de medicamentos. Assim, o estudo objetivou o alcance da totalidade, 42 sujeitos, da equipe de enfermagem do local de pesquisa, pois todos atuam diretamente na administração de medicamentos. No entanto, a amostra constitui-se de 35 profissionais: três enfermeiros, cinco técnicos em enfermagem e 27 auxiliares de enfermagem, uma vez que 7 auxiliares recusaram-se participar da pesquisa. Os enfermeiros fizeram parte desta pesquisa, uma vez que na instituição pesquisada, os mesmos administram diretamente as medicações. 
Os dados foram coletados a partir da utilização de um questionário, que foi adaptado de estudo consagrado pela literatura ${ }^{(15)}$. É importante salientar que às questões do referido estudo foi acrescentada a indagação: Quais as ações praticadas na instituição quando tais erros ocorreram? Esse questionário foi fornecido para os sujeitos da pesquisa, após explicação e esclarecimento de dúvidas pelo pesquisador. $\mathrm{O}$ questionário é compreendido como um conjunto de perguntas sobre um determinado tema, buscando medir a opinião, interesses, aspectos de personalidade e informação biográfica do respondente ${ }^{(16)}$.

Foi estipulado 15 dias para o preenchimento e devolução do instrumento de coleta de dados, ocorrendo de 16 de abril a 2 de maio/2008. O questionário contemplou os dados: sexo, faixa etária, categoria profissional, tempo de profissão, carga horária, turno e questões sobre identificação dos tipos de erros na administração de medicamentos, fatores que contribuíram para a ocorrência do erro e ações das instituições de saúde na ocorrência dos erros.

Os dados foram apresentados em forma de quadros, descritos por meio de porcentagens, e a discussão dos mesmos foi embasada em literatura nacional e internacional atualizadas.

O projeto de pesquisa teve a aprovação do Comitê de Ética em Pesquisa da Universidade Federal dos Vales do Jequitinhonha e Mucuri sob o processo de $n^{\circ} 007 / 08$, e o consentimento da direção da instituição hospitalar pesquisada. Os princípios éticos foram seguidos de acordo com a resolução 196/96 do Ministério da Saúde ${ }^{(17)}$.

\section{RESULTADOS E DISCUSSÃO}

Num total de 35 participantes, encontrou-se $94 \%$ do sexo feminino e 6\% do masculino. Quanto à faixa etária, 9\% estão entre 20 e 25 anos, 14\% entre 26 e 30 anos, 23\% entre 31 e 35 anos, 34\% entre 36 e 45 anos, $14 \%$ entre 46 e 50 anos e $6 \%$ entre 51 e 55 anos.

Em outro estudo que objetivou analisar o conhecimento da equipe de enfermagem acerca de medicamentos administrados em sua prática cotidiana, encontraram-se dados semelhantes a esses ${ }^{(18)}$.

Quanto à categoria profissional, 9\% são enfermeiros, $14 \%$ técnicos em enfermagem e $77 \%$ auxiliares de enfermagem. Constatou-se que 31\% possuem tempo de profissão entre 0 e 5 anos, 23\% entre 6 e 10 anos, 37\% entre 11 e 20 anos e 9\% entre 21 e 30 anos. Quanto à carga horária de trabalho, 94\% trabalham 48 horas por semana, 3\% 60 horas e 3\% 74 horas semanais. A respeito do turno de trabalho, 51\% pertencem ao diurno e $49 \%$ pertencem ao noturno, estando a literatura disponível sobre o tema, em concordância com os achados deste estudo ${ }^{(18)}$.

Quanto às questões referentes aos erros de medicação, quando questionado a respeito do número de erros cometidos em sua prática profissional desde quando começou a exercer a profissão, a soma de todos os erros corresponderam a 91 . Destes erros, $61 \%$ foram cometidos por auxiliares de enfermagem, 36\% por técnicos em enfermagem e 3\% por enfermeiros. Quanto a freqüência dos erros por turno de trabalho, 55\% ocorreram no período diurno e $45 \%$ no noturno.

$\mathrm{O}$ fato de acentuado número de erros ocorrerem no turno diurno pode estar relacionado à dinâmica de trabalho das instituições de saúde: neste período há uma maior quantidade de medicamentos a serem administrados e também é um horário de maior admissão de pacientes. Soma-se a isto, o fato do horário de visita ocorrer nesse turno, o que pode gerar um ambiente desfavorável às atividades da equipe de enfermagem ${ }^{(2)}$. Também a maior ocorrência de erros pelos técnicos e auxiliares de enfermagem, deve-se ao fato da administração de medicamentos ser praticada, na maioria das vezes, por esses profissionais ${ }^{(19)}$.

Os dados a seguir (Quadro 1) referem-se aos tipos de erros e aos fatores que contribuíram para a ocorrência dos mesmos.

Quadro 1 - Distribuição da ocorrência dos tipos de erros relacionados ao preparo e administração de medicação, 2008

\begin{tabular}{|lc|}
\hline Tipos de erros & Quantificação \% \\
Não monitoração do paciente após a & 22 \\
medicação & 19 \\
Via de administração errada & 17 \\
Diluição inadequada & \\
Medicamento administrado em paciente & 12 \\
errado & 12 \\
Não avaliação prévia do paciente & 11 \\
Dose errada & 07 \\
Medicamento errado & 100 \\
Total & \\
\hline
\end{tabular}

De acordo com o Quadro 1, a não monitoração do paciente após a medicação e a via de administração errada, correspondentes a $22 \%$ e $19 \%$ respectivamente, destacam-se como os principais tipos de erros na 
administração de medicamentos. A não monitoração do paciente após a medicação, surge como uma importante falha uma vez que os erros, muitas vezes, somente são identificados quando as conseqüências são clinicamente manifestadas pelo paciente, como a presença de sintomas ou reações adversas após algum tempo em que foi administrada a medicação ${ }^{(20)}$.

Os resultados alcançados nesse estudo revelam o despreparo da equipe de enfermagem em relação ao preparo e administração de medicamentos, o que pode levar a erros de cálculos, de preparo e de administração ${ }^{(21)}$, fatos que nos reportam à reflexão da importância do conhecimento técnico-científico da equipe de enfermagem e atualização constante deste conhecimento a respeito da prática de preparo e administração de medicamentos, sendo a educação em serviço um influenciador à redução da ocorrência de erros previsíveis.

Quanto à educação em serviço, destaca-se que os projetos devem estar em consonância com os interesses dos envolvidos, atender aos anseios e às necessidades daqueles que vão participar, aos objetivos da instituição e, no caso da enfermagem, à finalidade do trabalho, que é a maximização da assistência de enfermagem ${ }^{(22)}$, e propiciar à equipe conhecimentos sólidos sobre preparo e administração de medicamentos, exigidos pelo mercado de trabalho atual ${ }^{(23)}$.

A seguir, apresenta-se a descrição dos fatores que contribuíram para a ocorrência dos erros mencionados pelos sujeitos do estudo.

Quadro 2 - Distribuição dos fatores que contribuíram para a ocorrência dos erros relacionados ao preparo e administração das medicações, 2008

\begin{tabular}{|lc|}
\hline Fatores & Quantificação \% \\
Muitos pacientes/Excesso de trabalho & 25 \\
Poucos profissionais & 23 \\
Falta de atenção & 17 \\
Pouca experiência/Conhecimento & \\
insuficiente & 13 \\
Tumulto/Ambiente desfavorável & 6 \\
Cansaço/estresse & 6 \\
Prescrições inadequadas & 4 \\
Ansiedade do paciente & 2 \\
Falta de recursos físicos & 2 \\
Alteração de rotina & 2 \\
Total & 100 \\
\hline
\end{tabular}

No que se refere aos fatores contribuintes aos erros, destaca-se o fator "muitos pacientes/excesso de trabalho" com 25\% dos relatos, seguidos do fator "poucos profissionais", $23 \%$ e "falta de atenção", com $17 \%$.

O estudo identificou as más condições de trabalho da enfermagem (falta de profissionais, sobrecarga de trabalho, "cansaço/estresse" e "falta de atenção") como contribuintes para a ocorrência dos erros na prática da medicação, resultados que corroboram com estudos que afirmam que os erros são acentuados devido ao número insuficiente de profissionais para atender a alta demanda de cuidados $^{(21-24)}$. Com isso, fica clara a necessidade da reestruturação do serviço profissional com o fornecimento de um ambiente seguro e favorável ao desenvolvimento das atividades da equipe.

O conhecimento insuficiente e a pouca experiência também foram identificados como fatores contribuintes, o que aponta o fator humano como atributo das causas dos erros. Isso vai ao encontro dos dados de um estudo que aponta esse fator como causa comum das falhas ${ }^{(25)}$. Com isso a orientação voltada para esta questão se torna de fundamental importância, e, além disso, ressalta-se que cada profissional necessita buscar o seu próprio crescimento e ter consciência de que esse é falível.

No que concerne à questão referente à "pouca experiência” estudiosos do erro humano incluem os seguintes componentes: atos inseguros, enganos, falta de atenção, que apresentam maior incidência em indivíduos com pouca experiência ${ }^{(26)}$.

Outro aspecto levantado no estudo foi quanto às ações praticadas pela instituição frente aos erros na prática da medicação cometidos pelos funcionários. Somente 23 sujeitos responderam a essa pergunta e destes, 35\% relataram que a advertência é a ação mais praticada. A ausência de atitudes da instituição perante os erros foi mencionada por $26 \%$ da amostra. A orientação foi registrada com $22 \%$ e a advertência seguida de orientação com $17 \%$.

As advertências são vistas pelos indivíduos como uma forma de punição, e essa atitude possibilita medo, demissão, sentimento de culpa e preocupações relacionadas à gravidade do erro, o que pode levar os indivíduos envolvidos a não relatar seus erros e esse fato propiciar conseqüências devastadoras não só para os pacientes, como também para os profissionais envolvidos ${ }^{(12)}$.

A ausência de atitudes da instituição frente ao erro cometido pelo profissional, dado identificado neste 
estudo, também foi encontrado em outro que demonstrou a falta de estrutura de algumas instituições para lidar com tal ocorrência. Confirma-se também a resistência dessas instituições em admitir a existência do mesmo, o que faz com que não haja a definição nem a execução de estratégias para evitá-lo ${ }^{(27)}$, bem como uma avaliação mais reflexiva e criteriosa acerca de sua magnitude ${ }^{(24)}$.

Em vista disso, o National Coordinating Council for Medication Error Reporting and Prevention (NCCMERP), acredita que os profissionais da saúde e organizações devem ser incentivados a apresentar um relatório, buscando avaliar e prevenir os erros bem como a partilhar experiências com seus pares, devendose criar também uma cultura não punitiva, fornecer a confiabilidade adequada, proteções legais e propiciar a aprendizagem sobre erros e suas soluções aos profissionais ${ }^{(28)}$.

Destaca-se a importância da orientação em detrimento da advertência, visto que a orientação fornecerá capacitação e confiança para a realização do preparo e administração de medicamentos. Assim, a orientação contínua, bem como o aprimoramento da educação da equipe de enfermagem mostra-se como uma atitude de extrema importância, principalmente quando é voltada para o exame, diminuição/eliminação das dúvidas existentes anteriormente ao preparo e administração do medicamento(3). Essas orientações devem ser fornecidas pelo enfermeiro que necessita também realizar a supervisão como forma de minimizar os erros, sendo de extrema necessidade possuir amplo conhecimento na temática "administração de medicamentos”, buscando assim a confiança da sua equipe, uma prática mais humanizada e fundamentada cientificamente.

\section{CONSIDERAÇÕES FINAIS}

Com o presente estudo foi possível identificar os erros cometidos por profissionais da equipe de enfermagem, relacionados ao preparo e administração de medicamentos, bem como as ações praticadas pela instituição hospitalar em que os mesmos ocorreram. A identificação dos erros é sempre importante e esclarecedora, pois sustenta as decisões necessárias para evitá-los.

A presença de uma cultura punitiva perante o profissional que comete o erro, além de levar à subnotificação do mesmo, reflete que esta responsabilidade não é compartilhada entre o funcionário e a instituição que deveria prever e oferecer condições de trabalho para sua equipe.

Com isso fica evidente a necessidade de atitudes pessoais e institucionais, que buscam despertar a consciência de que o profissional é capaz de transformar sua realidade e de se tornar ponto chave na minimização dos erros e a garantia da confiança da equipe e do respeito aos pacientes.

Espera-se que este estudo contribua para alertar sobre a importância da qualidade da orientação que a equipe de enfermagem necessita receber para um preparo e administração de medicamentos mais seguros para os pacientes.

Esta qualidade é uma responsabilidade que deve ser compartilhada entre o enfermeiro e a instituição. É de fundamental importância que outras pesquisas sejam feitas para alertar e lançar luz sobre o tema, auxiliando as instituições e seus enfermeiros a fazerem o enfrentamento necessário e evitar as iatrogenias causadas, pela enfermagem, por erros de medicação.

\section{REFERÊNCIAS}

1. Nadzan DM. A System Approach to Medication Use. In: Cousins DM. Medication Use: Asystem Approach to Reducing Errors. Oakbrook Terrace: Joint Commission;1988.

2. Souza LO, Carvalho APS, Chianca TCM, Freitas ME, Ricaldoni CAC. Classificação de erros de medicação ocorridos em um hospital privado de Belo Horizonte. Rev Min Enferm. 2000;4(2):28.

3. Silva BK, Silva JS, Gobbo AFF, Miasso AI. Erros de medicação: condutas e propostas de prevenção na perspectiva da equipe de enfermagem. Rev Eletrônica Enfermagem [periódico na Internet]. 2007 [acesso em 2008Abril 11]9(3):712-23. Disponível em: www.fen.ufg.br.

4. Silva DO, Grou CR, Miasso AI, Cassiani SHB. Preparo e administração de medicamentos: análise de questionamentos e informações da equipe de enfermagem. Rev Latino-Am Enferm. 2007;15(5):1010-7.

5. Rothschild JM, Landrigan CP, Cronin JW, Kaushal R, Lockley SW, Burdick E, Stone PH, Lilly CM, Katz JT, Czeisler CA, Bates DW. The Critical Care Safety Study: The incidence and nature of adverse events and serious medical errors in intensive care. Crit Care Méd. 2005;33(8):1694-700.

6. Miasso AI, Grou CR, Cassiani SHB, Silva AEBC, Fakih FT. Erros de medicação: tipos, fatores causais e 
providências tomadas em quatro hospitais brasileiros. Rev Esc Enferm USP. 2006; 40(4):524-32.

7. Santos JO, Silva AEBC, Munari DB, Miasso AIi. Sentimentos de profissionais de enfermagem após a ocorrência de erros de medicação. Acta Paul Enferm. 2007;20(4):483-8.

8. Kopp BJ, Erstad BL, Allen ME, Theodorou AA, Priestley G. Medication errors and adverse drug events in an intensive care unit: direct observation approach for detection. Crit Care Med. 2006;34(2):415-25.

9. Knudsen P, Herborg H, Mortensen AR, Knudsen M, Hellebek A. Preventing medication errors in community pharmacy: frequency and seriousness of medication errors. Qual Saf Health Care. 2007;16(4):291-6.

10. Anselmi Ml, Peduzzi M, Dos Santos. Errors in the administration of intravenous medication in Brazilian hospitals. J Clin Nurs. 2007;16(10):1839-47.

11. Haw C, Stubbs J, Dickens G. An observational study of medication administration errors in old-age psychiatric inpatients. Int J Qual Health Care. 2007;19(4):210-6.

12. Schelbred AB, Nord R. Nurse's experiences of drug administration errors. J Advanc Nursing. 2007;60(3):317-24.

13. Richardson RJ. Pesquisa social: métodos e técnicas. $3^{\mathrm{a}}$ ed. São Paulo: Atlas; 2007.

14. Polit DF, Hungler BP. Nursing research: principles and methods. Philadelphia: Lippincott; 1999.

15. Carvalho VT, Cassiani SHB, Chiericato C. Erros mais comuns e fatores de risco na administração de medicamentos em unidades básicas de saúde. Rev Latino-Am Enferm. 1999; 7(5):67-75.

16. Yaremko RK, Harari H, Harrison RC, Lynn E. Handbook of research and quantitative methods in psychology. Hillsdale, NJ: Lawrence Erlbaum; 1986.

17. Brasil. Ministério da Saúde. Conselho Nacional de Saúde. Normas de pesquisa envolvendo seres humanos. Res. CNS 196/96. Bioética 1996; 4 Suppl:15-25.

18. Carvalho VT. Erros na administração de medicamentos: análise de relatos de profissionais de enfermagem [tese]. Ribeirão Preto (SP): Escola de Enfermagem de Ribeirão Preto. Universidade de São Paulo; 2000.

19. Grou CR, Cassiani SHB, Telles Filho PCP, Opitz SP.
Conhecimento de enfermeiras e técnicos de enfermagem em relação ao preparo e administração de medicamentos. Einstein. 2004; 2(3):182-6.

20. Carvalho VT, Cassiani SHB. Erros na medicação e conseqüências para profissionais de enfermagem e clientes: um estudo exploratório. Rev Latino-Am Enferm. 2002;10(4):523-9.

21. Monzani AAS. A ponta do iceberg: o método de notificação de erros de medicação em um hospital geral privado no município de Campinas-SP [dissertação]. Ribeirão Preto (SP): Escola de Enfermagem de Ribeirão Preto. Universidade de São Paulo; 2006.

22. Paschoal AS, Mantovani MF, Méier MJ. Percepção da educação permanente, continuada e em serviço para enfermeiros de um hospital de ensino. Rev Esc Enferm USP. 2007;41(3):478-84.

23. Telles Filho PCP, Cassiani SHB. Implementação e avaliação do módulo à distância "Administração de Medicamentos”. Cogitare Enferm. 2007;12(2):222-8.

24. Oliveira RC, Miasso AI, Cassiani SHB. Setor de emergência: situações de erros na medicação. In: Telles Filho PCP, Stuchi RAG, organizadores. A enfermagem no novo milênio: uma abordagem multidisciplinar. Belo Horizonte: Difusora; 2008. p.96-106.

25. Dibbi HM, Al-Abrashy HF, Hussain WA, Fatani MI, Karima TM. Causes and outcome of medication errors in hospitalized patients. Saudi Med J. 2006;27(10):148992.

26. Reason J. Human errors: models and management. British Medical J. 2000;320(7237):768-70.

27. Opitz SP. Sistema de medicação: análise dos erros nos processos de preparo e administração de medicamentos em um hospital de ensino [tese]. Ribeirão Preto (SP): Escola de Enfermagem de Ribeirão Preto. Universidade de São Paulo; 2006.

28. National Coordinating Council For Medication Error Reporting And Prevention-NCCMERP. Supports Principles For Patient Safety Reporting Programs, 2003. Disponível em: www.nccmerp.org/press/press2003-1125.html (14 abr. 2008). 